\title{
Patient-specific characterization of the invasiveness and proliferation of low-grade gliomas using serial MR imaging and a mathematical model of tumor growth
}

\author{
LEITH HATHOUT ${ }^{1}$, BENJAMIN M. ELLINGSON ${ }^{2-5}$, \\ TIMOTHY F. CLOUGHESY ${ }^{3,6}$ and WHITNEY B. POPE ${ }^{2}$
}

${ }^{1}$ Harvard Medical School, Boston, MA; ${ }^{2}$ Department of Radiological Sciences, ${ }^{3}$ UCLA Neuro-Oncology Program, and
${ }^{4}$ Department of Biomedical Physics, David Geffen School of Medicine, University of California, Los Angeles;
${ }^{5}$ Department of Bioengineering, Henry Samueli School of Engineering and Applied Science, University of California,
Los Angeles; ${ }^{6}$ Department of Neurology, David Geffen School of Medicine, University of California, Los Angeles, CA, USA

Received February 23, 2015; Accepted April 9, 2015

DOI: $10.3892 / o r .2015 .3926$

\begin{abstract}
Low-grade gliomas (LGGs) represent a significant proportion of hemispheric gliomas in adults. Although less aggressive than glioblastomas (GBMs), they have a broad range of biologic behavior, and often a limited prognosis. The aim of the present study was to explore LGG growth kinetics through a combination of routine MRI imaging and a novel adaptation of a mathematical tumor model. MRI imaging in 14 retrospectively identified grade II LGGs that showed some tumor enhancement was used to assess tumor radii at two separate time-points. This information was combined with a reaction-diffusion partial-differential equation model of tumor growth to calculate diffusion (D) and proliferation (Q) coefficients for each tumor, representing measures of tumor invasiveness and cellular multiplication, respectively. The results were compared to previously published data on GBMs. The average value of D was $0.034 \mathrm{~mm}^{2} /$ day and @ was $0.0056 /$ day. Grade II LGGs had a broad range of D and $\mathrm{Q}$. On average, the proliferation coefficient $\varrho$ was significantly lower than previously published values for GBM, by about an order of magnitude. The diffusion coefficient, modeling invasiveness, however, was only slightly lower but without statistical significance. It was possible to calculate detailed growth kinetic parameters for some LGGs, potentially providing a new way to assess tumor aggressiveness and possibly gauge prognosis. Even within a single-grade (WHO II), LGGs were found to have broad range of D and $\mathrm{Q}$, possibly correlating to their variable biologic behavior. Overall, the model parameters
\end{abstract}

Correspondence to: Dr Leith Hathout, Harvard Medical School, 107 Louis Pasteur Avenue, Boston, MA 02115-5750, USA

E-mail: leith_hathout@hms.harvard.edu

Abbreviations: LGG, low-grade glioma; GBM, glioblastoma

Key words: mathematical modeling, tumor biology, low-grade glioma suggest that LGG is less aggressive than GBM based primarily on a lower index of tumor proliferation rather than on lesser invasiveness.

\section{Introduction}

Low-grade (WHO grade II) gliomas (LGGs) represent $\sim 25$ to $30 \%$ of hemispheric gliomas in adults (1). Despite the absence of gross mitoses, nuclear pleomorphism and vascular endothelial proliferation (i.e., the histologic features which characterize high-grade gliomas), they nonetheless have a somewhat poor prognosis, with median survival rates of $7-8$ years $(2,3)$. This is, in large measure, due to the diffusely infiltrative growth pattern of these tumors, which gradually invade the adjacent neuropil $(4,5)$. On the one hand, this invasive growth pattern stands in distinction to that of the 'benign' or non-infiltrative astrocytic (WHO grade I) tumors, such as pilocytic astrocytoma, but on the other, it is significantly less pronounced than the capacity for invasion and proliferation exhibited by high-grade gliomas such as glioblastoma (GBM) (6). Within these vague boundaries, there seems to be a broad range of growth kinetics for LGGs. Indeed, it is generally recognized that tumor grading is not precise, as the characteristics of invasion and proliferation actually represent a biological continuum among astrocytomas (7). This continuum exists not only between grades, but also within them, as the proliferative index of grade II gliomas has been shown to vary among tumors, tending, for example, to be higher in older patients (8).

This broad variability of growth characteristics, the relatively young age of patients at presentation, and the potential morbidity of therapies, have led to considerable controversy over the optimal management of LGGs. This controversy emerges from what some authors have termed the dilemma between 'too much therapy, too early and too little, too late' $(9,10)$. One approach to navigating the dilemma lies in the concept which Duffau has termed 'the fundamental principle of personalized management' (11). Among the emerging tools to help realize this goal of personalized tumor management is the measurement of individual in vivo tumor kinetics using 
a combination of mathematical modeling and MR imaging. This approach allows the use of routine MR imaging data in a novel and quantitative fashion to characterize tumor growth in individual patients.

The combination of mathematical modeling with tumor imaging has already been successfully used to model the growth kinetics of GBM by some investigators. It was originally pioneered by Harpold etal(12), Tracqui etal(13), Wang etal(14), and Murray (15), using a reaction-diffusion partial-differential equation model of cell density as a function of space and time. The model accounts for both the infiltrative nature of the tumor using a diffusion term, and the net proliferation of tumor cells using a proliferation term (12-15). To date, however, there have been only limited applications of this model to low-grade gliomas $(16,17)$.

This paper introduces the reaction-diffusion model, and applies it to a population of low-grade gliomas to calculate both their diffusion and proliferation coefficients, with several important results. To the knowledge of the authors, this is the first such detailed application of this model to LGGs.

\section{Materials and methods}

Mathematical model. The mathematical model relies on a reaction-diffusion partial-differential equation which calculates cell density as a function of space and time. The model uses a mass-balance approach, stating that in any given location, the number of tumor cells increases only by new cells which move into the region or new cells produced by tumor proliferation. Thus, the model accounts for both the infiltrative nature of the tumor using a diffusion term, and the proliferation of tumor cells using a proliferation term (12-15). The proliferation of the tumor is modeled in standard fashion using a so-called logistic growth term, where-in the rate of growth slows down as the cell density increases toward the tissue carrying capacity (12).

In words, the equation of the tumor model can be stated as follows:

Rate of change of tumor cell density (at a location $\mathrm{x}$ ) $=$ Net invasion (diffusion) of tumor cells + net proliferation of tumor cells. In mathematical terms, this may be restated as (12):

$$
\frac{\partial c(t, x)}{d t}=\nabla \bullet(D(x) \nabla c)+\rho c\left(1-\frac{c}{K}\right)
$$

where the various terms are defined as follows: $c(t, x)$ is the tumor cell density, in terms of cells $/ \mathrm{mm}^{3}$, which is a function of position $\mathrm{x}$ and time t. $\mathrm{D}(\mathrm{x})$ is the diffusion term, in $\mathrm{mm}^{2} /$ day, which models local tumor invasion of tumor cells $\varrho c\left(1-\frac{\mathrm{c}}{\mathrm{K}}\right)$ is a logistic tumor growth term, where $\mathrm{Q}$ is the tumor proliferation rate in units of (/day), governed by a tissue tumor carrying capacity $\mathrm{K}$, in units of cells $/ \mathrm{mm}^{3}$.

With a constant diffusion term, and in one spatial dimension, we get the classical Fisher-Kolmogorov reaction-diffusion equation successfully used by previous investigators to model many aspects of GBM growth dynamics $(12,13,15)$ :

$$
\frac{\partial c}{d t}=D \frac{\partial^{2} c}{d x^{2}}+\rho c\left(1-\frac{c}{K}\right) \quad \text { Equation (1) }
$$

The differential equation is solved using a standard finite-difference algorithm in Matlab.
The initial and boundary conditions are similar to those conventionally employed in the reaction diffusion models of Rockne et al (18). Specifically, we used $c(0, x)=0.8 K * e^{-0.25 x^{2}}$. The carrying capacity for the tissue $\mathrm{K}$, can be considered a cell density of $10^{5}$ cells $/ \mathrm{mm}^{3}$ (19). The solution domain is $\mathrm{L}=200 \mathrm{~mm}$, and we use the standard zero-flux boundary conditions $\frac{\partial c}{d x}=0$ at $\mathrm{x}=0$ and at $\mathrm{x}=\mathrm{L}(20)$.

The above reaction-diffusion model provides an evolution of the tumor cell density with time, where cell density gradually increases toward the carrying capacity $\mathrm{K}$ and where the tumor grows in space, with the tumor cell-density curve moving to the right (Fig. 1).

As can be seen from Fig. 1, at any time-point, the model predicts a gradient of cell density, decreasing with distance from the center of the tumor. These mathematical predictions mirror direct histological observations of cell density gradients in GBM (21). The model estimates this gradient by setting cell density thresholds for tumor visibility on both $\mathrm{T} 1$ post-contrast images and FLAIR/T2 images (12,22) (Fig. 2).

In addition, using $\mathrm{T} 1$ post-contrast tumor radii from two serial MRI scans, a radial velocity of the tumor is calculated. The estimated tumor cell density gradient and the radial tumor velocity can then be used to calculate individual values of $D$ and $\varrho$ for each patient, as described by Harpold et al (12), and used by Swanson et al $(14,22,23)$.

It is noted that using Equation 1, it is possible to mathematically derive an asymptotic estimate of the rate of radial tumor growth (i.e., tumor velocity) over long growth times. This estimate, known as the Fisher's approximation, states that $v=2 \sqrt{D \rho}(14,18,22)$.

Patient selection, MR imaging and tumor measurements. Fourteen patients with grade II glioma were retrospectively identified from the UCLA Neuro-Oncology Database from January 2005 to January 2013 who had a biopsy proven diagnosis of non-transforming grade II LGG, but with some contrast enhancement. Data acquisition was performed in compliance with all applicable regulations of the Health Insurance Portability and Accountability Act. All UCLA patients in this study signed institutional review board-approved informed consent to have their data included in our research database.

Standard MR imaging data were acquired by using either a 1.5T or 3T MR imaging scanner (Sonata/Avanto/Trio/Verio; Siemens, Erlangen, Germany) using pulse sequences supplied by the manufacturer. Typical imaging sets included axial T1-weighted (TR, $400 \mathrm{msec}$; TE, $15 \mathrm{msec}$; section thickness, $5 \mathrm{~mm}$ ), T2-weighted fast spin-echo (TR, 4,000 msec; TE, 126-130 msec; section thickness, $5 \mathrm{~mm}$ ), and FLAIR (TR, 8,802 msec; TE, $122 \mathrm{msec}$; TI, 2,100 msec; section thickness, $3 \mathrm{~mm}$ ) and gadopentetate dimeglumine enhanced (Magnevist, $0.1 \mathrm{mmol} / \mathrm{kg}$; Berlex, Wayne, NY, USA) axial and coronal T1-weighted images (TR, $400 \mathrm{msec}$; TE, $15 \mathrm{msec}$; section thickness, $3 \mathrm{~mm}$ ), with an FOV of $24 \mathrm{~cm}$ and a matrix size of 256x256. Post-contrast images were acquired immediately following contrast injection.

Contrast-enhancing and T2-weighted hyperintense tumor regions were segmented by using standard techniques. Briefly, tumor ROIs were isolated by manually defining the relative region of tumor occurrence, thresholding post-contrast T1-weighted images within these regions by using an empiric 

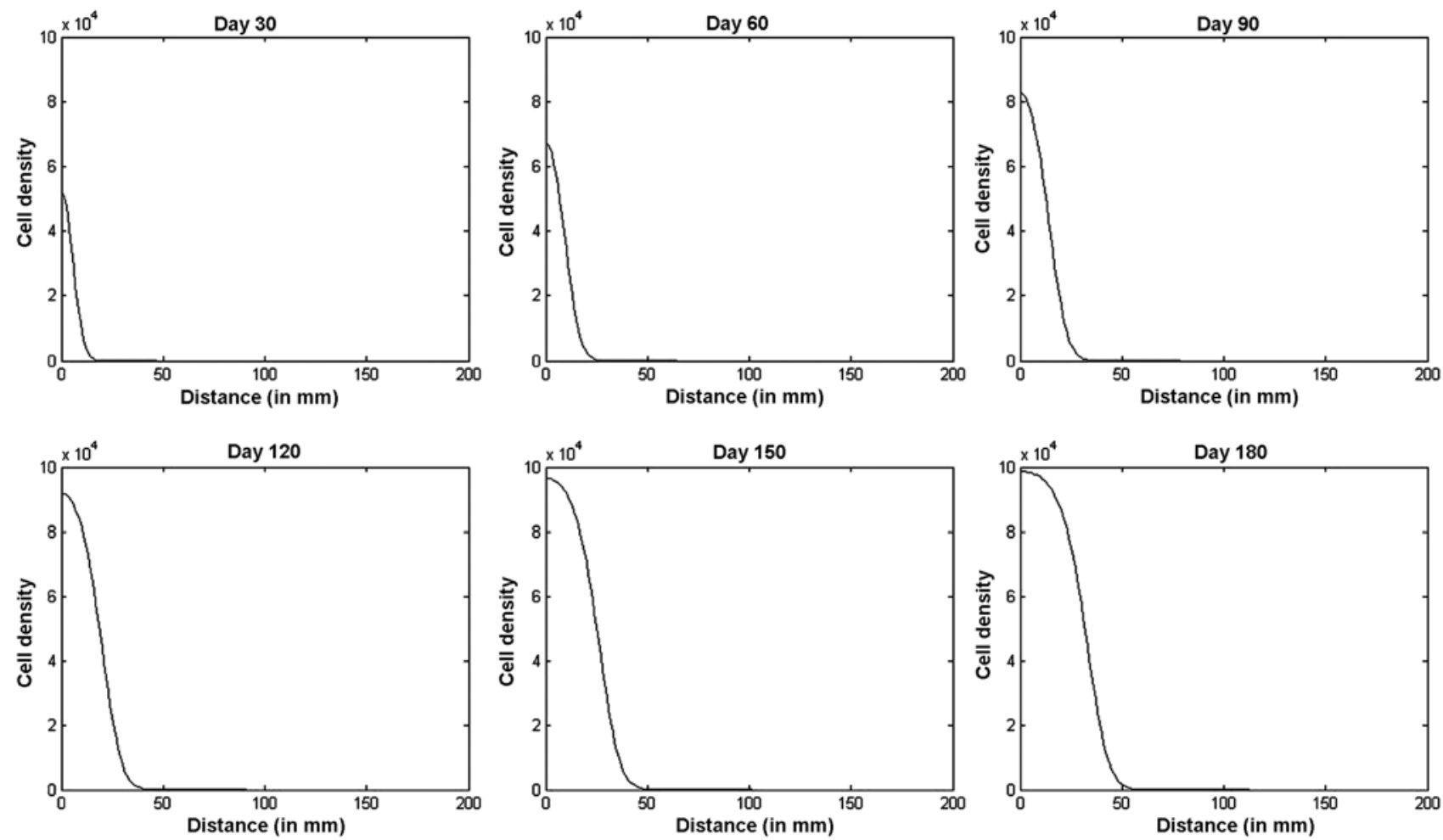

Figure 1 . The time evolution of the cell density profile as given by the standard reaction-diffusion model of Equation 1 , with $\mathrm{K}=10^{5} \mathrm{cells} / \mathrm{mm}^{3}, \mathrm{D}(0.4 \mathrm{~mm} /$ day) and $\varrho(0.04 /$ day $)$.

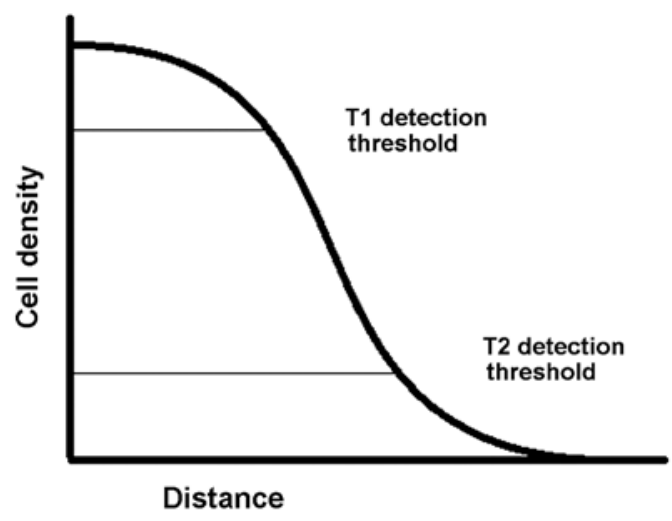

Figure 2. Schematic showing cell density vs. distance from tumor center, illustrating the use of cell density thresholds to delineate calculated tumor radius on $\mathrm{T} 1$ post-contrast and $\mathrm{T} 2$-weighted images.

threshold, and then manually editing the resulting masks to exclude any non-tumor tissue. Tumor volume (V) was calculated, and mean tumor radius was calculated as:

$$
r=\sqrt[3]{\frac{3 V}{4 \pi}}
$$

This was performed on two MRI scans separated in time, with the number of days tallied between each scan to allow calculation of tumor velocity in terms of $\mathrm{mm} /$ day (Fig. 3).

\section{Results}

As described above, D and $\mathrm{Q}$ were calculated from the mathematical model, with results shown in Table I. These values give individual estimates of tumor invasiveness and net proliferation for each patient. It is noted that there was a significant spread in values for both D and Q. For the LGG cohort, the average value of $\mathrm{D}$ was $0.0338 \mathrm{~mm}^{2} /$ day, and the average value of $\varrho$ was $0.00564 /$ day.

Although there was significant variation in the values of D and $\mathrm{Q}$, it is useful to also look at both individual and average values for the entire cohort in relation to more aggressive tumors, such as GBM, to give a sense of the relative behavior of LGGs to GBMs in terms of invasiveness and proliferation.

Data for D and $\mathrm{Q}$ have been previously published in Wang et al for 32 GBMs (14). This was used for comparison with our cohort of LGGs. The data show that GBMs have, on average, higher diffusion and proliferation coefficients than LGGs. In terms of $\mathrm{D}$, the average for the full cohort of GBMs was $0.078 \mathrm{~mm}^{2} /$ day, vs. 0.0338 for the cohort of LGGs $(\mathrm{P}=0.125$ for a two-tailed t-test). In terms of $\varrho$, the difference was much more significant, with an average $\mathrm{Q}$ of $0.089 /$ day for GBMs, compared to $0.00564 /$ day for LGGs $(\mathrm{P}=0.0035$ for a twotailed t-test).

For the purposes of illustration, Fig. 4 shows a graphical display of D and $\mathrm{Q}$ for GBMs vs. LGGs, using 13 GBMs chosen at random from the cohort published in Wang et al (14).

The data show that GBMs, on average, have higher diffusion (D) and proliferation (@) rates, with the difference in proliferation coefficients showing high statistical significance (see Discussion).

\section{Discussion}

The initial management of patients with low-grade gliomas typically involves observation with serial MR imaging, 


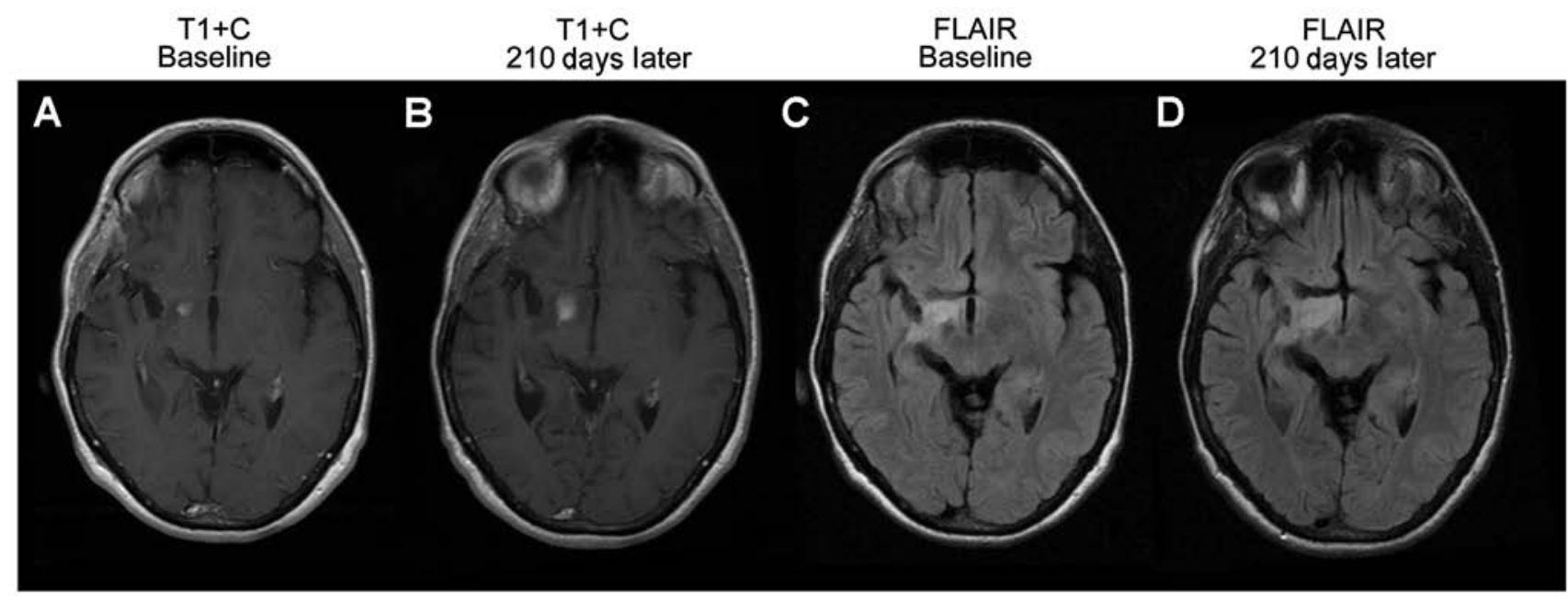

Figure 3. Post-contrast T1 axial (A and B) and FLAIR axial (C and D) images in a representative patient separated by 210 days.

Table I. Calculated values for D and $\varrho$ for each of the tumor patients.

\begin{tabular}{lll}
\hline Patient & $\mathrm{D}\left(\mathrm{mm}^{2} / \mathrm{day}\right)$ & $\varrho$ (per day $)$ \\
\hline 1 & 0.00248994 & 0.00022432 \\
2 & 0.00682525 & 0.00070728 \\
3 & 0.00454081 & 0.00042162 \\
4 & 0.00505126 & 0.00287003 \\
5 & 0.00138135 & 0.01491739 \\
6 & 0.02763844 & 0.00453089 \\
7 & 0.00935318 & 0.00696439 \\
8 & 0.10542301 & 0.03073557 \\
9 & 0.00451929 & 0.00305978 \\
10 & 0.02943589 & 0.00434799 \\
11 & 0.04582795 & 0.00303496 \\
12 & 0.03063511 & 0.00033665 \\
13 & 0.04600428 & 0.00232697 \\
14 & 0.01689673 & 0.00152223 \\
\hline
\end{tabular}

D, tumor diffusion coefficient; $\varrho$, tumor diffusion coefficient.

usually with qualitative or semi-quantitative data (i.e., overall tumor size) used to help guide therapy. This study presents a novel combination of routine MR imaging and mathematical modeling to extract more precise quantitative information about tumor growth kinetics. Specifically, the use of a reaction-diffusion partial-differential equation model of tumor growth and two serial MR scans was sufficient to allow estimates of tumor invasiveness, modeled by D (the tumor diffusion coefficient), and tumor proliferation, modeled by $\varrho$ (the tumor proliferation coefficient). This represents, to the knowledge of the authors, the first such estimates for LGGs, and potentially represents a substantial new extension in characterizing their biologic behavior.

As a first assessment of the model's results, the diffusion and proliferation coefficients for our LGG population were compared to values for $32 \mathrm{GBMs}$ previously published in

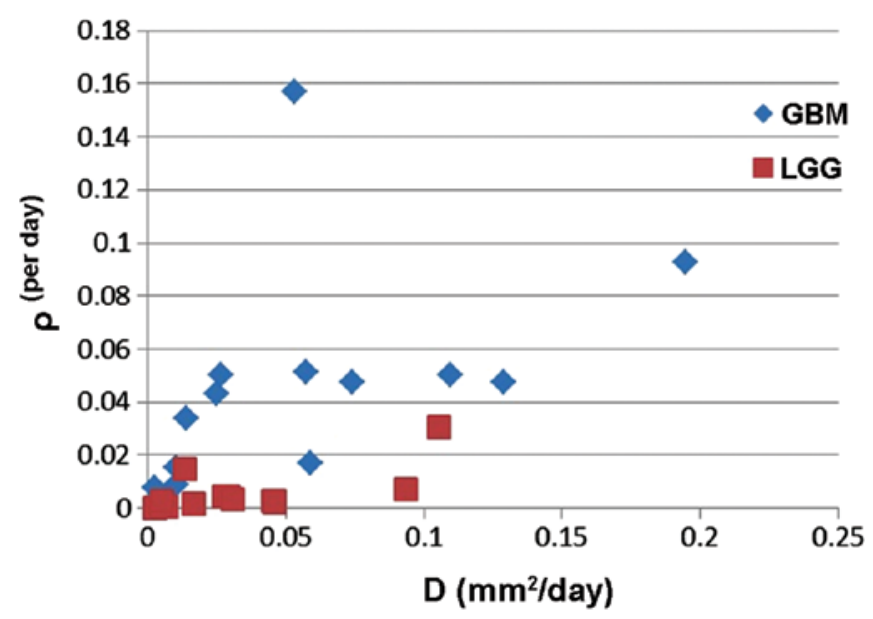

Figure 4. D and $\varrho$ plot for LGGs vs. GBMs. The LGGs have on average lower D and lower . LGGs, low-grade gliomas; GBMs, glioblastomas; D, tumor diffusion coefficient; $\varrho$, tumor proliferation coefficient.

Wang et al (14). This comparison shows that there is a difference in both diffusion and proliferation coefficients, with GBMs exhibiting higher coefficients, as expected. A notable outcome is that the difference between LGGs and GBMs is much more pronounced in the proliferation coefficient $\varrho$ than in D. These results represent the first empiric testing of the assumptions made by Woodward et al in their modeling of the efficacy of surgery for gliomas, specifically that LGGs have diffusion and proliferation rates that are about one-tenth that of GBMs (12). Our results suggest that the assumption is true for proliferation, but that the difference is much less pronounced for diffusion. This, in turn, suggests that for our model's parameters, the aggressiveness of GBMs relies more on their high tumor proliferation rates than on greater local invasiveness, although both are significant factors.

Most importantly, though, the model shows a significant heterogeneity of the diffusion and proliferation coefficients among the LGG cohort, underscoring the broad range of growth characteristics within a group of tumors all classified as grade II gliomas. Indeed, earlier quantitative study has demonstrated the broad heterogeneity which exists among 
low-grade gliomas. For example, a recent study by Pallud et al estimated the velocity of diametric tumor expansion for a large group of LGGs, finding a range of $5.8 \pm 6.3 \mathrm{~mm} /$ year (26). In that study, it was shown that the velocity of spontaneous tumor expansion is quite variable among LGGs, and is a strong predictor of survival, independent of tumor genetics (26). This is concordant with our model, since high tumor velocities correlate directly with high D and high $\mathrm{Q}$.

Thus, to date, however, most kinetics study on LGGs has focused on the aggregate parameter of tumor growth rate (corresponding to radial tumor velocity $\mathrm{v}$ in our model). This approach has generated some important results. The study of Pallud et al showed that higher tumor velocities correlated with more aggressive tumors and shorter survival times (26). More recently, Brasil Caseiras et al demonstrated that a 6-month tumor velocity was the single best predictor of the likelihood and timing of malignant transformation, which had previously been considered a fairly unpredictable event (27).

However, as the Fisher approximation $v=2 \sqrt{D \rho}$ shows, the same radial velocity can arise from various combinations of $\mathrm{D}$ and $\mathrm{Q}$. The main contribution of the present study is that it allows individual delineation of $\mathrm{D}$ and $\mathrm{Q}$, which is a significant refinement over using just the radial tumor velocity, and which may have significant prognostic and therapeutic implications.

The separate effect of D and $\varrho$ on such issues as time to progression to high-grade glioma, and on patient survival, is thus suggested as a significant avenue of future research. Such an approach may have the capacity to refine the important results of Pallud et al and Brasil Caseiras et al $(26,27)$. For example, looking at the LGG vs. GBM D and $\varrho$ data, it is noted that $\mathrm{Q}$ differs significantly more than $\mathrm{D}$ between the 2 groups, with about a 2-fold difference in $\mathrm{D}$, but slightly greater than an order of magnitude difference in $\varrho$. This raises the possibility that in LGGs, small variations in D could have more impact than small variations in $\varrho$ for the same tumor velocity. On the other hand, it suggests that larger tumor growth velocities, such as the fast growing tumors in the LGG cohorts studied by Pallud et al and Brasil Caseiras et al, likely arise from significantly larger values of $\mathrm{Q}$ rather than D.

These issues, of course, need to be settled empirically. However, the model suggests that D and @ are likely to have an individual significance in tumor biologic behavior beyond their aggregate contribution to velocity of growth. This can be seen by considering the $\mathrm{D} / \mathrm{Q}$ ratio, which has been termed the 'invisibility index' of the tumor. This ratio correlates to the proportion of tumor cells which are beyond the T2/FLAIR tumor margin (12). The phenomenon of tumor infiltration beyond the MR-visible tumor margins is well-documented, and is significant to patient prognosis (28). In our cohort of grade II LGGs, there was a wide spectrum of $\mathrm{D} / \mathrm{Q}$ ratios, ranging from 0.93 to 19.8 , showing that it is an important factor to consider. Our simulations show that in terms of the model, a D/Q ratio of 1 correlates with $1.8 \%$ of tumor cells being beyond the $\mathrm{T} 2$ boundary, while for a D/Q ratio of $30,5.3 \%$ of cells are beyond the $\mathrm{T} 2$ boundary. The $\mathrm{D} / \mathrm{Q}$ ratio also has a direct correlate to the distance which cells are present beyond the visible tumor margin, this distance being greater for tumors with a high $\mathrm{D} / \mathrm{Q}$ ratio; this, in turn, may have a significant impact on the success or failure of surgical resection. The importance of the D/Q ratio to the accurate prediction of survival of GBM patients, using the above model and incorporating the extent of surgical resection, has already been preliminarily demonstrated (23). Conversely, a low D/@ ratio suggests relatively aggressive tumor proliferation, and low $\mathrm{D} / \mathrm{Q}$ ratios have been shown by Szeto et al to be associated with higher levels of tumor hypoxia using FIMSO-PET (29). This higher tissue hypoxia, in turn, may correlate with radioresistance.

Clearly, mathematical models are idealizations of a much more complex underlying biology, but they have utility if they can capture essential features of the phenomenon under study. As noted above, this study presents a new application of the reaction-diffusion model to LGGs, showing the model's utility, and pointing to avenues of future research.

The present iteration of the model has several limitations. In general, of course, there is cellular heterogeneity within all tumors, and hence calculation of parameters such as D and @ represents an aggregate measure. Another limitation is that invasiveness is an active process, and can only be roughly modeled as a passive diffusion phenomenon. These limitations would apply to all gliomas. More specific to the present model and its adaptation from GBMs to LGGs is the need for contrast enhancement within the tumor for the calculation of $\mathrm{D}$ and $\mathrm{Q}$. This restricts the application of the model, since only a minority of LGGs show contrast enhancement. Although figures of up to $40 \%$ have been quoted in the literature, that may be an overestimate $(30,31)$. Our group is currently investigating the possibility of extending the model to also analyze the kinetics of non-enhancing gliomas. This may require an analysis of linear growth velocities and the use of the Fisher's approximation, in conjunction with a priori average estimates of $\mathrm{D}$ in order to calculate $\mathrm{Q}$. Given the fact that there is only a 2-fold difference in D between LGGs and GBMs, it may not be unreasonable to assume a relative stability of $\mathrm{D}$ between enhancing and non-enhancing LGGs. A similar approach, assuming an average value of $\varrho$ for GBMs, has been used by Swanson et al to model patient survival after surgery (23). However, the accuracy of such approaches will require further empiric confirmation.

In conclusion, a reaction-diffusion partial-differential equation model, previously used in GBM modeling, can be applied to low-grade gliomas. Using only routine MRI scans at 2 time-points, individual estimates of tumor invasiveness and tumor proliferation may be obtained. This model may explain variability in aggressiveness of low-grade gliomas as well as significant differences in growth kinetics compared to GBM.

\section{References}

1. Russell DS and Rubinstein LJ (eds): Pathology of Tumors of the Nervous System. 5th edition. Williams and Wilkins, Baltimore, 1989.

2. Vertosick FT Jr, Selker RG and Arena VC: Survival of patients with well-differentiated astrocytomas diagnosed in the era of computed tomography. Neurosurgery 28: 496-501, 1991.

3. McCormack BM, Miller DC, Budzilovich GN, Voorhees GJ and Ransohoff J: Treatment and survival of low-grade astrocytoma in adults - 1977-1988. Neurosurgery 31: 636-642, 1992.

4. van den Bent MJ, Afra D, de Witte O, Ben Hassel M, Schraub S, Hoang-Xuan K, Malmström PO, Collette L, Piérart M, Mirimanoff R, et al; EORTC Radiotherapy and Brain Tumor Groups and the UK Medical Research Council: Long-term efficacy of early versus delayed radiotherapy for low-grade astrocytoma and oligodendroglioma in adults: the EORTC 22845 randomised trial. Lancet 366: 985-990, 2005. 
5. Mandonnet E, Delattre JY, Tanguy ML, Swanson KR, Carpentier AF, Duffau H, Cornu P, Van Effenterre R, Alvord EC Jr and Capelle L: Continuous growth of mean tumor diameter in a subset of grade II gliomas. Ann Neurol 53: 524-528, 2003.

6. VandenBerg SR: Current diagnostic concepts of astrocytic tumors. J Neuropathol Exp Neurol 51: 644-657, 1992.

7. Burger PC and Scheithauer BW (eds): Atlas of Tumor Pathology, Tumors of the Central Nervous System. 3rd Series, Fascicle 10. Armed Forces Institute of Pathology, Washington, DC, 1994.

8. Shafqat S, Hedley-Whyte ET and Henson JW: Age-dependent rate of anaplastic transformation in low-grade astrocytoma. Neurology 52: 867-869, 1999.

9. Soffietti R, Baumert BG, Bello L, von Deimling A, Duffau H, Frénay M, Grisold W, Grant R, Graus F, Hoang-Xuan K, et al; European Federation of Neurological Societies: Guidelines on management of low-grade gliomas: report of an EFNS-EANO Task Force. Eur J Neurol 17: 1124-1133, 2010.

10. Ruda R, Bertero L and Soffietti R: Natural history and spontaneous prognostic factors. Chapter 18. In: Diffuse Low-Grade Gliomas in Adults: Natural History, Interaction with the Brain and New Individualized Therapeutic Strategies. Duffau H (ed). Springer, London, 2013.

11. Duffau H (ed): Diffuse Low-Grade Gliomas in Adults: Natural History, Interaction with the Brain and New Individualized Therapeutic Strategies. Springer, London, p2, 2013.

12. Harpold HL, Alvord EC Jr and Swanson KR: The evolution of mathematical modeling of glioma proliferation and invasion. J Neuropathol Exp Neurol 66: 1-9, 2007.

13. Tracqui P, Cruywagen GC, Woodward DE, Bartoo GT, Murray JD and Alvord EC Jr: A mathematical model of glioma growth: the effect of chemotherapy on spatio-temporal growth. Cell Prolif 28: 17-31, 1995.

14. Wang CH, Rockhill JK, Mrugala M, Peacock DL, Lai A, Jusenius K, Wardlaw JM, Cloughesy T, Spence AM, Rockne R, et al: Prognostic significance of growth kinetics in newly diagnosed glioblastomas revealed by combining serial imaging with a novel biomathematical model. Cancer Res 69: 9133-9140, 2009.

15. Murray JD: Growth and control of brain tumors. Chapter 11 In: Mathematical Biology II: Spatial Models and Biomedical Applications. Springer, New York, pp536-613, 2003.

16. Mandonnet E, Pallud J, Clatz O, Taillandier L, Konukoglu E, Duffau $\mathrm{H}$ and Capelle L: Computational modeling of the WHO grade II glioma dynamics: principles and applications to management paradigm. Neurosurg Rev 31: 263-269, 2008.

17. Pallud J, Taillandier L, Capelle L, Fontaine D, Peyre M, Ducray F, Duffau H and Mandonnet E: Quantitative morphological magnetic resonance imaging follow-up of low-grade glioma: a plea for systematic measurement of growth rates. Neurosurgery 71: 729-740, 2012

18. Rockne R, Rockhill JK, Mrugala M, Spence AM, Kalet I, Hendrickson K, Lai A, Cloughesy T, Alvord EC Jr and Swanson KR: Predicting the efficacy of radiotherapy in individual glioblastoma patients in vivo: a mathematical modeling approach. Phys Med Biol 55: 3271-3285, 2010.
19. Herculano-Houzel S and Lent R: Isotropic fractionator: a simple, rapid method for the quantification of total cell and neuron numbers in the brain. J Neurosci 25: 2518-2521, 2005.

20. Rockne R, Alvord EC Jr, Rockhill JK and Swanson KR: A mathematical model for brain tumor response to radiation therapy. J Math Biol 58: 561-578, 2009.

21. Swanson KR and Alvord EC Jr: Serial imaging observations and postmortem examination of an untreated glioblastoma: a traveling wave of glioma growth and invasion. Neuro Oncol 4: $340,2002$.

22. Corwin D, Holdsworth C, Rockne RC, Trister AD, Mrugala MM, Rockhill JK, Stewart RD, Phillips M and Swanson KR: Toward patient-specific, biologically optimized radiation therapy plans for the treatment of glioblastoma. PLoS One 8: e79115, 2013

23. Swanson KR, Rostomily RC and Alvord EC Jr: A mathematical modelling tool for predicting survival of individual patients following resection of glioblastoma: a proof of principle. Br J Cancer 98: 113-119, 2008

24. Burger PC, Heinz ER, Shibata T and Kleihues P: Topographic anatomy and CT correlations in the untreated glioblastoma multiforme. J Neurosurg 68: 698-704, 1988

25. Concannon JP, Kramer S and Berry R: The extent of intracranial gliomata at autopsy and its relationship to techniques used in radiation therapy of brain tumors. Am J Roentgenol Radium Ther Nucl Med 84: 99-107, 1960.

26. Pallud J, Blonski M, Mandonnet E, Audureau E, Fontaine D, Sanai N, Bauchet L, Peruzzi P, Frénay M, Colin P, et al: Velocity of tumor spontaneous expansion predicts long-term outcomes for diffuse low-grade gliomas. Neuro Oncol 15: 595-606, 2013.

27. Brasil Caseiras G, Ciccarelli O, Altmann DR, Benton CE, Tozer DJ, Tofts PS, Yousry TA, Rees J, Waldman AD and Jäger HR: Low-grade gliomas: six-month tumor growth predicts patient outcome better than admission tumor volume, relative cerebral blood volume, and apparent diffusion coefficient. Radiology 253: 505-512, 2009.

28. Price SJ, Jena R, Burnet NG, Hutchinson PJ, Dean AF, Peña A, Pickard JD, Carpenter TA and Gillard JH: Improved delineation of glioma margins and regions of infiltration with the use of diffusion tensor imaging: an image-guided biopsy study. AJNR Am J Neuroradiol 27: 1969-1974, 2006.

29. Szeto MD, Chakraborty G, Hadley J, Rockne R, Muzi M, Alvord EC Jr, Krohn KA, Spence AM and Swanson KR: Quantitative metrics of net proliferation and invasion link biological aggressiveness assessed by MRI with hypoxia assessed by FMISO-PET in newly diagnosed glioblastomas. Cancer Res 69: 4502-4509, 2009.

30. Osborn AG (ed): Diagnostic Neuroradiology. Mosby, St. Louis, 1994.

31. Atlas SW, Lavi E and Fisher PG: Intraaxial brain tumors. In: Magnetic Resonance Imaging of the Brain and Spine. Atlas SW (ed). Lippincott, Williams and Wilkins, Philadelphia, pp565-693, 2002. 